\title{
Mathematical aspects of Rietveld refinement and crystal structure studies on $\mathrm{PbTiO}_{3}$ ceramics
}

\author{
NIRANJAN SAHU* and S PANIGRAHI \\ Department of Physics, National Institute of Technology, Rourkela 769 008, India
}

MS received 12 January 2010; revised 29 October 2010

\begin{abstract}
The core mathematics, goodness-of-fit parameters of Rietveld refinement technique is introduced for structural analysis of crystalline materials not available as single crystals. X-ray diffraction (XRD) patterns of $\mathrm{PbTiO}_{3}$ compound prepared by following solid-state route, suggests it to be in single crystal form. All the observed peaks could be indexed to $P 4 \mathrm{~mm}$ space group with tetragonal symmetry. XRD pattern is analysed by employing Rietveld method. The unit cell parameters are found to be $a=b=3.8987(0.0008) \AA$ and $c=4.1380(0.0009) \AA$. The axial ratio $c / a$ and unit cell volume are found to be 1.0614 and $62.896(0.023) \AA^{3}$. Bond lengths and angles are calculated using the cell parameters. Using the Rietveld refinement parameters a stable $\mathrm{PbTiO}_{3}$ structure is suggested.
\end{abstract}

Keywords. Rietveld; $\mathrm{PbTiO}_{3}$; XRD; oxides.

\section{Introduction}

Rietveld refinement technique is a standard treatment of powder diffraction data to make the final structural model or otherwise it is a procedure for structural solution in nature. This method has the ability to extract detailed crystal structural information from powder diffraction data. In Rietveld method the least-square refinements are carried out until the best fit is obtained between the entire observed powder diffraction pattern taken as a whole and the entire calculated pattern based on the simultaneously refined models for the crystal structure(s), diffraction optic effects, instrumental factors and other specimen characteristics (e.g. lattice parameters) as may be desired (Young and Wiles 1982).

Lead titanate $\left(\mathrm{PbTiO}_{3}, \mathrm{PT}\right)$ has drawn considerable interest in fundamental research due to its rich physical properties. This material shows high Curie temperature and possesses high spontaneous polarization. It is a ferroelectric material that has a Curie temperature of $490^{\circ} \mathrm{C}$ and displays pyroelectric and piezoelectric properties (Ishikawa et al 1988; Ramesh and Schlom 2002; Zembilgotov et al 2002; Fu and Bellaiche 2003; Fong et al 2004; Jiang et al 2004; Lang and Jiang 2007; Zhang et al 2008). This material is being considered for applications in different kinds of devices, such as storage information, piezoelectric actuators, infrared sensors and ultrasonic transducers in medical and solar applications (Obmann and Galaubitt 1997; Kim et al 1999; Iakovlev et al 2004). Recently, lots of efforts have been put into prepare bulk

\footnotetext{
*Author for correspondence (nsahu76@gmail.com)
}

as well as thin film of $\mathrm{PbTiO}_{3}$ (Venkateswarlu et al 2005; Sahu et al 2010). However, a large tetragonality ( $c / a=$ 1.063) of tetragonal phase PT resulting in a high stress within the lattice leads to a frangibility and creates difficulty in the preparation of corresponding ceramics (Jona and Shirane 1993). The isomorphic substitution of lead with lanthanum gives rise to decrease in the tetragonality, and the material with a formula of $\mathrm{Pb}_{0.7} \mathrm{La}_{0.3} \mathrm{TiO}_{3}$ (PLT30) has a pseudo cubic perovskite structure (Wójcik 1989; Tickoo et al 2002; Neves et al 2004). However, according to our knowledge, detailed structural analysis for $\mathrm{PbTiO}_{3}$ prepared at different temperatures and method is lagging to sort out the synthesis of stable $\mathrm{PbTiO}_{3}$. We have prepared PT by solid-state route to study its crystal structure. The detailed crystal structure has been studied by analysing XRD pattern recorded at room temperature using Rietveld method and FULLPROF software (Young et al 1996).

Rietveld (1988) provided an account of the inception and early development of this method and of the growth of its acceptance from being initially ignored to the very wide spread and vigorous use in many fields. In this paper, we provide a general introductory account and core mathematics of Rietveld refinement. Using the refinement parameter a stable $\mathrm{PbTiO}_{3}$ crystal structure is suggested.

\section{Core mathematics and goodness of fit parameters of Rietveld refinement}

A powder diffraction pattern can be recorded in numerical form for a discrete set of scattering angles, times of 
flight or energies. The Rietveld mathematical model is to describe the full powder diffraction profile, fundamentals of the least-squares optimization for crystallographic applications and the nature of the different kinds of free parameters in the crystallographic and profile models (Prince 1982). Hence the diffraction pattern is recorded in digitized form i.e. as a numerical intensity value, at each of several thousand equal increments (steps), in the pattern. Depending on the method, the increments may be either in scattering angle, $2 \theta$, or some energy parameter such as velocity (for time-of-flight neutron data) or wavelength (for X-ray data collected with an energy dispersive detector and an incident beam of 'white' X-radiation). For constant wavelength data, the increments are usually steps in scattering angle and the intensity $y_{i}$ at each step ' $i$ ', in the pattern is measured either directly with a quantum detector on a diffractometer or indirectly with stepscanning micro densitometry of film (e.g. Guinier) data (Guinier 1963). Typical step sizes range from 0.01 to $0 \cdot 05^{\circ} 2 \theta$ for fixed wavelength X-ray data.

In all cases, the 'best-fit' sought is the best least squares fit to all of the thousand of $y_{i}$ 's simultaneously. The quantity minimized in the least-squares refinement:

$$
S_{y}=\sum_{i} w_{i}\left(y_{i o}-y_{i c}\right)^{2} ; w_{i}=1 / y_{i},
$$

where $y_{i}$ is the observed intensity at the $i$ th step, $Y_{c i}$ the calculated intensity at the $i$ th step and the sum is overall data points.

A powder diffraction pattern of a crystalline material may be thought of as a collection of individual reflection profiles, each of which has a peak height, a peak position, breadth, tails which decay gradually with distance from the peak position and an integrated area which is proportional to the Bragg intensity, $I_{k}$, where $k$ stands for the Miller indices, $h, k, l . I_{k}$ is proportional to the square of the absolute value of the structure factor, $\left|F_{k}\right|^{2}$. In all powder diffraction patterns, Rietveld method is not needed in the first place, these profiles are not all resolved but partially overlap one another to a substantial degree. It is a crucial feature of the Rietveld method that no effort is made in advance to allocate observed intensity to particular Bragg reflections or to resolve overlapped reflections. Consequently, a reasonably good starting model is needed. The method is a structure refinement method (Prince 1985).

Typically, many Bragg reflections contribute to the intensity, $y_{i}$, observed at any arbitrarily chosen point, $I$, in the pattern. The calculated intensities $y_{c i}$, are determined from $\left|F_{k}\right|^{2}$ values calculated from the structural model by summing of the calculated contributions from neighbouring (i.e. within a specified range) Bragg reflections plus the background

$$
y_{c i}=S \Sigma_{k} L_{k}\left|F_{k}\right|^{2} \phi\left(2 \theta_{i}-2 \theta_{k}\right) P_{k} A+y_{b i},
$$

where $S$ is the scale factor, $k$ represents the Miller indices, $h k l$ for a Bragg's reflection, $L_{k}$ contains the Lorentz, polarization, and multiplicity factors, $\phi$ the reflection profile function, $P_{k}$ is the preferred orientation function, $A$ is an absorption factor, $F_{k}$ is the structure factor for $k$ th Bragg reflection and $Y_{b i}$ is the background intensity at $i$ th step.

The least squares minimization procedures lead to a set of normal equations involving derivatives of all of the calculated intensities, $y_{c i}$, with respect to each adjustable parameter and are soluble by inversion of the normal matrix with elements, $M_{j k}$ formally given by

$$
M_{j k}=\Sigma 2 w_{i}\left[\left(y_{i}-y_{c i}\right) \partial^{2} y_{c i} / \partial^{2} x_{j} \partial x_{k}-\left(\partial y_{c i} / \partial x_{j}\right)\left(\partial y_{c i} / \partial x_{k}\right)\right],
$$

where the parameters $x_{j}, x_{k}$ are the (same set of) adjustable parameters. In the use of this algorithm, it is a common practice to approximate these matrix elements by deletion of the first term, which is $\left(y_{i}-y_{c i}\right)$.

One is, thus, dealing with the creation and inversion of an $M$ by $M$ matrix, where $M$ is the number of parameters being refined. Because the residual function is non-linear, the solution must be found with an iterative procedure in which the shifts, $\Delta x_{k}$, are

$$
\Delta x_{k}=\Sigma\left(M_{j k}-1\right) \partial s_{y} / \partial x_{k} .
$$

The calculated shifts are applied to the initial parameters to produce an improved model and the whole procedure is then repeated. Because the relationships between the adjustable parameters and the intensities are non-linear, the starting model must be close to the correct model or the non-linear least squares procedure will not lead to the global minimum (Rietveld 1988). The model parameters that may be refined include not only atomic position, thermal and site-occupancy parameters but also parameters for the background, lattice, instrumental geometrical-optical features, specimen aberrations (e.g. specimen displacement and transparency), an amorphous component and specimen reflection-profile-broadening agents such as crystallite and microstrain.

For angle dispersive data, the dependence of the breadth $H$ of the reflection profiles measured as fullwidth-at-half-maximum (FWHM) has typically been modeled as Caglioti et al (1958).

$$
\Gamma_{k}^{2}=H^{2}=U \tan ^{2} \theta_{k}+V \tan \theta_{k}+W
$$

where $U, V$ and $W$ are the refinable parameters.

Because preferred orientation arises when there is a stronger tendency for the crystallites in a specimen to be oriented more one way, or one set of ways, than all others. Because preferred orientation produces systematic distortions of the reflection intensities. The distortions can be mathematically modeled with 'preferred orientation functions', $P_{k}$ in (2). $P_{k}$ was implemented in Rietveld's own early program. Here $G_{1}$ and $G_{2}$ are refinable para- 
meters and $\alpha_{k}$ is the angle between $d_{k}$ and the fibre axis direction. This $P_{k}$ is useful if the degree of preferred orientation is not large. When this function is used in Rietveld refinements with data from a standard $\theta-2 \theta$ $\mathrm{X}$-ray diffractometer, the refined parameter changes sign.

$$
\begin{aligned}
& P_{k}=\exp \left(-G_{1} \alpha_{k}^{2}\right), \\
& P_{k}=G_{2}+\left(1-G_{2}\right) \exp \left(-G_{1} \sin ^{2} \alpha_{k}\right), \\
& P_{k}=G_{2}+\left(1-G_{2}\right) \exp \left(-G_{1} \sin ^{3} \alpha_{k}\right) .
\end{aligned}
$$

Dollase (1986) showed superior performance of the March function. Many authors have now incorporated the March-Dollase function in their Rietveld refinement codes and confirm Dollase's evaluation, (Dollase 1986). One of the particular merits of this preferred orientation function is

$$
P_{k}=\left(G_{1}^{2} \cos ^{2} \alpha_{k}+\frac{1}{G_{1}} \sin ^{2} \alpha_{k}\right)^{-3 / 2}
$$

Another interesting and apparently very powerful preferred-orientation function is that of Ahtee et al (1989). The preferred orientation effect is modeled by expanding the orientation distribution in spherical harmonics. They have incorporated the preferred orientation model in their new Rietveld refinement code in which they have also used a true Voigt function for the reflection profile function. They state that 'the code is applicable to neutron, synchrotron and conventional X-ray data when the incident radiation is essentially one wavelength'. It would seem that those who find cases in which the MarchDollase approach does not give satisfactory results might consider using this more complex but, in principle, more powerful approach.

The structure factor $F_{k}$ is given by

$$
\begin{aligned}
& F_{k}=\sum_{j=1}^{n} N_{j} f_{j} \exp \left(2 \pi i h_{k}^{t} r_{j}-2 \pi^{2} h_{k}^{t} B_{j} h_{k}\right), \\
& F_{k}=\sum_{j=1}^{n} N_{j} f_{j} \exp \left[2 \pi i\left(h x_{j}+k y_{j}+\ell z_{j}\right)\right] \exp \left[-B_{j}\right], \\
& B_{j}=8 \pi^{2} u^{2} \sin ^{2} \theta / \lambda^{2},
\end{aligned}
$$

where $h, k$ and $l$ are the Miller indices, $x_{j}, y_{j}$ and $z_{j}$ are the position parameters of the $j$ th atom in the unit cell. $B_{j}$ is the scattering length, $u^{2}$ is the root-mean-square thermal displacement of the $j$ th atom parallel to the diffraction vector and $N_{j}$ the site occupancy multiplier for the $j$ th atom site in (11),

$$
\begin{aligned}
F_{k}= & \exp \left(-8 \pi^{2}\left\langle u^{2}\right\rangle \frac{\sin ^{2} \theta}{\lambda^{2}}\right) \\
& \times \sum_{j=1}^{n} N_{j} f_{j} \exp \left[2 \pi i\left(h x_{j}+k y_{j}+\ell z_{j}\right)\right], \\
& f=\sum_{i=1}^{4} a_{i} \exp \left(-b_{i} \frac{\sin ^{2} \theta}{\lambda^{2}}\right)+c+\Delta f^{\prime}+\Delta f^{\prime \prime} .
\end{aligned}
$$

The above equation (12) represents atomic scattering factor, where $a, b, c$ are from the 'International Tables for Crystallography'. $\Delta f^{\prime}$ and $\Delta f^{\prime \prime}$, must be checked and changed for synchrotron radiation (Wiles and Young 1981). Examination of (2) and (9) can help one to appreciate the complementary relationship between Rietveld structural refinements done with X-ray data and those done with neutron data. The only really significant difference arises in the $f_{j}$ factor in (9), which, then, is inserted into (2).

For this, we set aside magnetic scattering of neutrons and deal only with nuclear scattering, which takes place by short-range nuclear forces. X-rays are scattered, almost only by the electron surrounding the nuclei. Thus, the region from which they are scattered, which is about 1-2 $\AA$ in diameter, is of the order of $10^{4}$ larger than the region from which the neutrons scattered. In each case, the scattering factor has the form of the Fourier transform (Guinier 1963) of the distribution of scattering density (density of the scattering material times its scattering power). The result is that the X-ray scattering factor falls off seriously over the range of $(\sin \theta) / \lambda$ in which one can make observations with the commonly used wavelengths in the range $0 \cdot 5-2 \cdot 5 \AA$.

In this paper, the basic equations concerned with the calculation performed in FULLPROF are discussed. It is supposed that the reader has a good knowledge of powder diffraction, so the explanations given below concern mainly the description of the terms involved in the formulae. The quality of the agreement between observed and calculated profiles is measured by a set of conventional factor. In FULLPROF two sets of indices are calculated, according to the meaning of the integer ' $n$ '. In the first set, ' $n$ ' is the total number of points used in the refinement $(n=$ NPTS-NEXC $=$ total number of points in the pattern minus total number of excluded points) (Marshall and Lovesey 1971). In the second set, only those points where there is Bragg's contribution are taken into account. The definition of the indices and some oftenlyused numerical criteria of fit are as follows

$$
R_{p}=\frac{\sum_{i}\left|y_{i o}-y_{i c}\right|}{\sum_{i} y_{i o}},
$$




$$
\begin{aligned}
& R_{\exp }=\left[\frac{N-P}{\sum_{i} w_{i} y_{i o}^{2}}\right]^{1 / 2}, \\
& R_{B}=\frac{\sum_{i}\left|I_{k o}-I_{k c}\right|}{\sum_{i} I_{k o}}, \\
& R_{w p}=\left[\frac{\sum_{i} w_{i}\left(y_{i o}-y_{i c}\right)^{2}}{\sum_{i} w_{i} y_{i o}^{2}}\right]^{1 / 2}, \\
& \mathrm{GOF}=\frac{\sum_{i} w_{i}\left(y_{i o}-y_{i c}\right)^{2}}{N-P}=\left(\frac{R_{w p}}{R_{\exp }}\right)^{2}=S^{2},
\end{aligned}
$$

where $N-P$ is the number of degrees of freedom. The meaning of ' $N$ ' has been given above and $P$ is the number of refined parameters. The experimental points within the excluded regions are always excluded from the calculation of all agreement factors (Rotella 1988).

\section{Experimental}

$\mathrm{PbTiO}_{3}$ compound was prepared by the following solidstate route. Stoichiometric ratio of $\mathrm{Pb}\left(\mathrm{NO}_{3}\right)_{2}$ and $\mathrm{TiO}_{2}$ with $99.9 \%$ purity were weighed by using a high precision electronic balance. The above materials were mixed thoroughly with the help of agate mortar and pestle. The grinding was carried out under acetone till the acetone evaporated from the mortar. The mixture was presintered at $600^{\circ} \mathrm{C}$ for $36 \mathrm{~h}$ with intermediate grindings. The sintering in pellet form was carried out at $1000^{\circ} \mathrm{C}$ for over $40 \mathrm{~h}$ with intermediate grindings and repelletizing. All the above sintering processes were carried out in air. The detailed flow diagram for the preparation of $\mathrm{PbTiO}_{3}$ is given in figure 1 . The scanning electron micrograph was recorded using LEO SEM. The compositional SEM EDS carried out the analysis. XRD pattern at room temperature for the sample was recorded by using Bruker D-8 Advance XRD machine. The $\mathrm{CuK} \alpha$ radiation was used as $\mathrm{X}$-ray source. The machine was operated at $40 \mathrm{kV}$ and $40 \mathrm{~mA}$. The data was collected with a step size of $0 \cdot 01^{\circ}$ and time constant of $1 \mathrm{~s}$.

\section{Results and discussion}

XRD patterns were recorded at room temperature using a BRUKER D-8 ADVANCE XRD machine by employing $\mathrm{CuK} \alpha$ radiation. The XRD patterns recorded for $\mathrm{PbTiO}_{3}$ compound is shown in figure 2. One can see that the sample is essentially in single phase form within the instrumental error. All the observed peaks could be indexed to $P 4 \mathrm{~mm}$ space group with tetragonal symmetry.

The XRD pattern was refined by Rietveld method and by using the FULLPROF program. The refined XRD pattern is shown in figure 3 . All the observed peaks of single

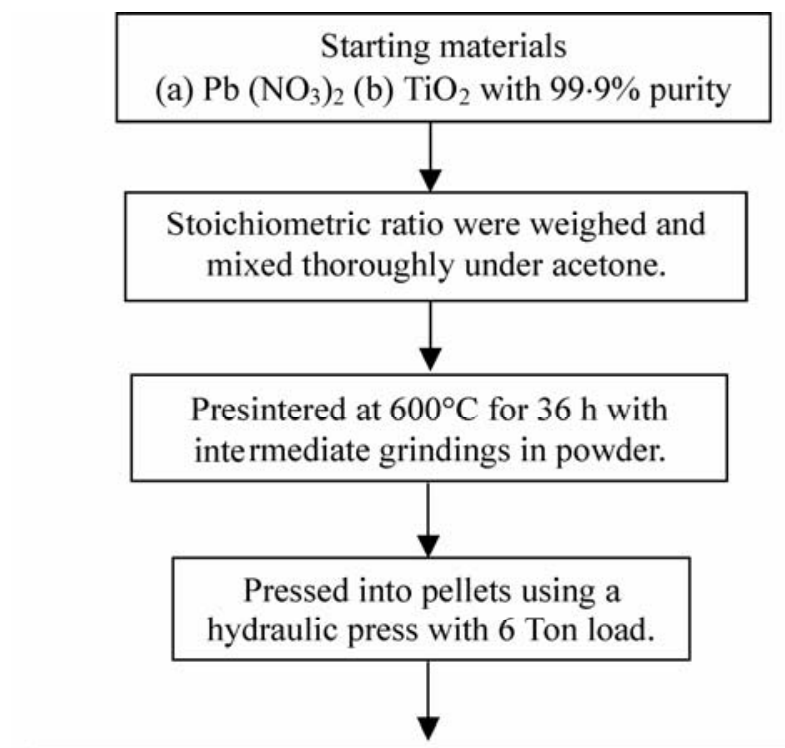

Sintering in pellet form $\rightarrow 1000^{\circ} \mathrm{C}$ for over $40 \mathrm{~h}$ with intermediate grindings and repelletising

All the above sintering processes were carried out in air.

Figure 1. Flow diagram for processing of PT ceramics with powders prepared by solid-state route.

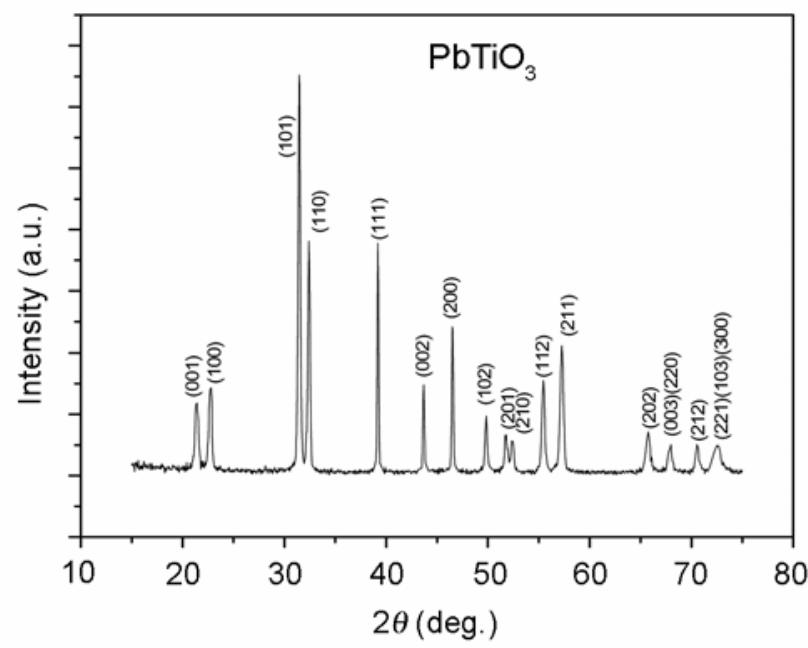

Figure 2. XRD pattern of $\mathrm{PbTiO}_{3}$. Observed peaks are indexed to $\mathrm{P} 4 \mathrm{~mm}$ space group. 
phase $\mathrm{PbTiO}_{3}$ compound prepared by following the solidstate route could be indexed to $P 4 \mathrm{~mm}$ space group with tetragonal symmetry. The pseudo-Voigt function was chosen for the profile shape refinement because it is used to resolve strain and size contribution to peak broadening. The $P V(x)$ (Pseudo Voigt) function is a linear combination of a Lorentzian $L^{\prime}$ and a Gaussian $G^{\prime}$ of the same $\operatorname{FWHM}\left(H^{2}\right)$. So there are two parameters characterizing the peak shape: $P V(x)=P V(x, \eta, H)$. If the $L^{\prime}(x)$ and $G^{\prime}(x)$ are normalized, $P V(x)$ is also normalized. The integral breadth of a normalized Pseudo-Voigt function is just the inverse of the maximum value. The FWHM is same for $L(x), G(x)$ and $P V(x)$ (Young 1996). Allowing the variation of different parameters such as cell parameters,

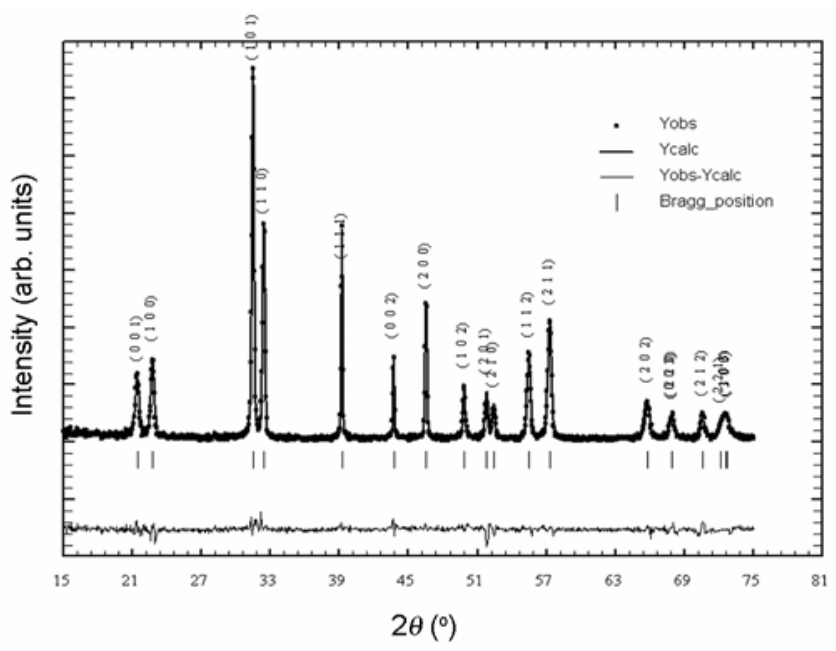

Figure 3. XRD pattern for $\mathrm{PbTiO}_{3}$ with refined data obtained by Rietveld method. Experimental points are given as (.) and theoretical data are shown as solid line. Difference between theoretical and experimental data is shown as bottom line. The vertical lines represent the Bragg's allowed peaks.

Table 1. Position parameters of $\mathrm{Pb}, \mathrm{Ti}, \mathrm{O}_{1}$ and $\mathrm{O}_{2}$.

\begin{tabular}{lccc}
\hline Atom & $X$ & $Y$ & $Z$ \\
\hline $\mathrm{Pb}$ & $0 \cdot 00000$ & $0 \cdot 00000$ & 0.00000 \\
$\mathrm{Ti}$ & $0 \cdot 50000$ & 0.50000 & 0.53000 \\
$\mathrm{O} 1$ & $0 \cdot 50000$ & $0 \cdot 50000$ & 0.07410 \\
$\mathrm{O} 2$ & 0.50000 & $0 \cdot 00000$ & 0.64090 \\
\hline
\end{tabular}

Table 2. Bond length using the parameters obtained from Rietveld analysis.

\begin{tabular}{lcccc}
\hline No. & Atom 1 & Atom 2 & Distance & Quantity \\
\hline 1 & $\mathrm{~Pb}$ & $\mathrm{Ti}$ & 3.5228 & 4 \\
2 & $\mathrm{Ti}$ & $\mathrm{O}_{1}$ & 1.8865 & 1 \\
3 & $\mathrm{Ti}$ & $\mathrm{O}_{2}$ & $2 \cdot 0026$ & 4 \\
4 & $\mathrm{Ti}$ & $\mathrm{Pb}$ & 3.3738 & 4 \\
5 & $\mathrm{O}_{2}$ & $\mathrm{~Pb}$ & $2 \cdot 4511$ & 8 \\
\hline
\end{tabular}

scale factors, position parameters and isotropic thermal parameters carried out the refinement. The position of the $\mathrm{Pb}$, Ti and $\mathrm{O}$ atoms are given in table 1. Two oxygen positions were taken for the refinement. The occupancy was taken as fixed as it is a lighter atom; hence it is not very sensitive to XRD. It is difficult to refine the oxygen occupancy from XRD data.

The refined cell parameters are found to be $a=b=$ $3.8987(0.0008) \AA$ and $c=4 \cdot 1380(0.0009) \AA$. These values are found to be comparable to those in Jona and Shirane (1993). The unit cell volume is found to be 62.896 (0.023) $\AA$. The goodness-of-fit parameters are found to be $R_{\mathrm{p}}=3 \cdot 70, \quad R_{\mathrm{wp}}=4 \cdot 20, \quad R_{\exp }=3.91, \quad R_{\mathrm{Bragg}}=$ $5.63, R_{\mathrm{f}}=3.20$ and $\chi^{2}=1 \cdot 32$. The axial ratio $c / a$ is found to be 1.0614 , which is less than that of earlier reported value of 1.063 (Jona and Shirane 1993). The low axial value shows that the material is more compact and structure is more stable. Hence preparation of low axial ratio compound by following solid-state route is thus reported here.

The bond lengths and angles were calculated from the refined data with the help of POWDER CELL Program. The average axial and in plane bond lengths are found to be 1.8865 and $2.0026 \AA$, respectively. All the bond lengths are listed in table 2 . These values are found to be comparable with those reported in the literature. All the calculated bond angles are listed in table 3 and these are comparable with those of earlier reported values. The structure of the compound is shown in figure 4. This structure has been drawn using the refined parameters.

\section{Conclusions}

It has been most gratifying for us to experience how the Rietveld method has contributed to a renewed interest in

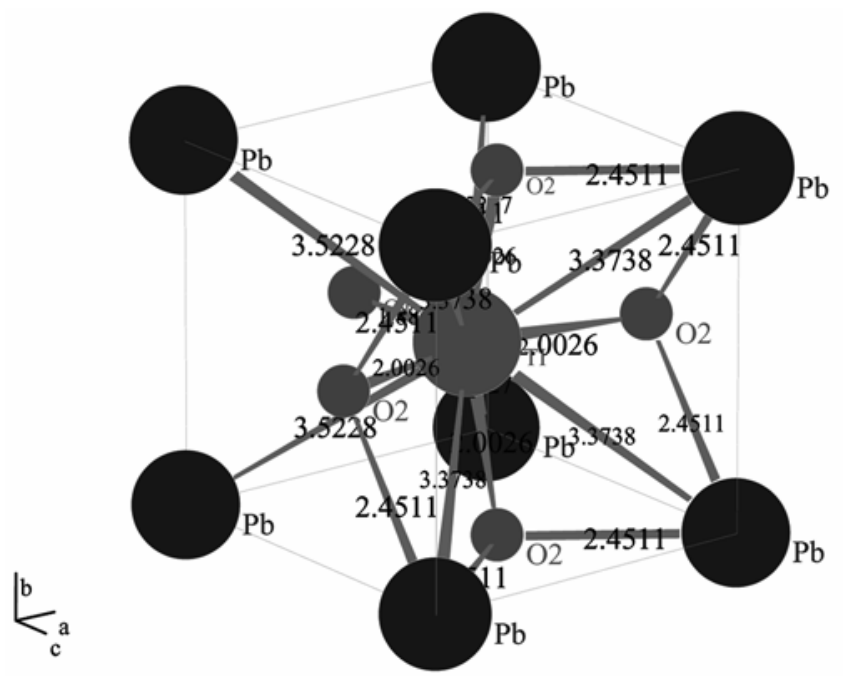

Figure 4. Structure of $\mathrm{PbTiO}_{3}$ obtained from Rietveld analysis parameters. 
Table 3. Bond angles calculated using the parameters obtained from Rietveld analysis.

\begin{tabular}{|c|c|c|c|c|}
\hline Atom 1 & Atom 2 & Atom 3 & Angle & Quantity \\
\hline $\mathrm{Pb}$ & $\mathrm{Ti}$ & $\mathrm{O}_{1}$ & 51.4957 & 3 \\
\hline $\mathrm{Pb}$ & $\mathrm{Ti}$ & $\mathrm{O}_{2}$ & $66 \cdot 6733$ & 6 \\
\hline $\mathrm{Pb}$ & $\mathrm{Ti}$ & $\mathrm{Pb}$ & $67 \cdot 1950$ & 4 \\
\hline $\mathrm{Pb}$ & $\mathrm{Ti}$ & $\mathrm{O}_{2}$ & 132.9454 & 6 \\
\hline $\mathrm{Pb}$ & $\mathrm{Ti}$ & $\mathrm{Pb}$ & 102.9922 & 2 \\
\hline $\mathrm{Pb}$ & $\mathrm{Ti}$ & $\mathrm{Pb}$ & $73 \cdot 7068$ & 2 \\
\hline $\mathrm{Pb}$ & $\mathrm{Ti}$ & $\mathrm{Pb}$ & $111 \cdot 0323$ & 8 \\
\hline $\mathrm{Pb}$ & $\mathrm{Ti}$ & $\mathrm{Pb}$ & $176 \cdot 6986$ & 1 \\
\hline $\mathrm{O}_{1}$ & $\mathrm{Ti}$ & $\mathrm{O}_{2}$ & $103 \cdot 2468$ & 2 \\
\hline $\mathrm{O}_{1}$ & $\mathrm{Ti}$ & $\mathrm{O}_{2}$ & $103 \cdot 2477$ & 2 \\
\hline $\mathrm{O}_{1}$ & $\mathrm{Ti}$ & $\mathrm{Pb}$ & $51 \cdot 4965$ & 1 \\
\hline $\mathrm{O}_{1}$ & $\mathrm{Ti}$ & $\mathrm{Pb}$ & $125 \cdot 2020$ & 1 \\
\hline $\mathrm{O}_{1}$ & $\mathrm{Ti}$ & $\mathrm{Pb}$ & $125 \cdot 2026$ & 3 \\
\hline $\mathrm{O}_{2}$ & $\mathrm{Ti}$ & $\mathrm{O}_{2}$ & 86.9897 & 3 \\
\hline $\mathrm{O}_{2}$ & $\mathrm{Ti}$ & $\mathrm{Pb}$ & $132 \cdot 9445$ & 2 \\
\hline $\mathrm{O}_{2}$ & $\mathrm{Ti}$ & $\mathrm{O}_{2}$ & 153.5056 & 2 \\
\hline $\mathrm{O}_{2}$ & $\mathrm{Ti}$ & $\mathrm{Pb}$ & $46 \cdot 0113$ & 6 \\
\hline $\mathrm{O}_{2}$ & $\mathrm{Ti}$ & $\mathrm{Pb}$ & $46 \cdot 0119$ & 2 \\
\hline $\mathrm{O}_{2}$ & $\mathrm{Ti}$ & $\mathrm{Pb}$ & $115 \cdot 4878$ & 6 \\
\hline $\mathrm{Ti}$ & $\mathrm{O}_{2}$ & $\mathrm{~Pb}$ & $97 \cdot 9855$ & 6 \\
\hline $\mathrm{Pb}$ & $\mathrm{Ti}$ & $\mathrm{O}_{2}$ & $66 \cdot 6742$ & 2 \\
\hline $\mathrm{Pb}$ & $\mathrm{Ti}$ & $\mathrm{Pb}$ & $73 \cdot 7063$ & 2 \\
\hline $\mathrm{Pb}$ & $\mathrm{Ti}$ & $\mathrm{Pb}$ & $176 \cdot 6984$ & 3 \\
\hline $\mathrm{O}_{2}$ & $\mathrm{Ti}$ & $\mathrm{O}_{2}$ & $86 \cdot 9893$ & 1 \\
\hline $\mathrm{O}_{2}$ & $\mathrm{Ti}$ & $\mathrm{Pb}$ & $115 \cdot 4871$ & 2 \\
\hline $\mathrm{Ti}$ & $\mathrm{O}_{2}$ & $\mathrm{~Pb}$ & $97 \cdot 9861$ & 2 \\
\hline $\mathrm{Pb}$ & $\mathrm{Ti}$ & $\mathrm{Pb}$ & $70 \cdot 5908$ & 2 \\
\hline $\mathrm{Pb}$ & $\mathrm{Ti}$ & $\mathrm{Pb}$ & $109 \cdot 5952$ & 2 \\
\hline $\mathrm{Ti}$ & $\mathrm{Pb}$ & $\mathrm{O}_{2}$ & $36 \cdot 0032$ & 8 \\
\hline $\mathrm{Pb}$ & $\mathrm{Ti}$ & $\mathrm{Pb}$ & $70 \cdot 5904$ & 2 \\
\hline $\mathrm{Pb}$ & $\mathrm{O}_{2}$ & $\mathrm{~Pb}$ & $105 \cdot 3639$ & 4 \\
\hline $\mathrm{O}_{2}$ & $\mathrm{~Pb}$ & $\mathrm{O}_{2}$ & $68 \cdot 4369$ & 3 \\
\hline $\mathrm{O}_{2}$ & $\mathrm{~Pb}$ & $\mathrm{O}_{2}$ & $68 \cdot 4363$ & 1 \\
\hline
\end{tabular}

powder diffraction techniques. The formulae have been given for the inclusion of absorption, multiple scattering and extinction in Rietveld analysis. In core mathematics the agreement factor is included. We have prepared a single phase $\mathrm{PbTiO}_{3}$ compound by following the solidstate route. All the observed peaks could be indexed to $P 4 m m$ space group with tetragonal symmetry. From Rietveld analysis the unit cell parameters are found to be $a=b=3.8987(0.0008) \AA$ and $c=4.1380(0.0009) \AA$. The axial ratio $c / a$ is found to be 1.0614 , which is less than that of reported value of 1.0630 . The average axial and in plane bond lengths are found to be 1.8865 and $2.0026 \AA$, respectively. Finally, using the Rietveld refinement parameters, a stable $\mathrm{PbTiO}_{3}$ structure was obtained.

\section{References}

Ahtee M, Nurmela M, Suortii P and Jarvinen M 1989 J. Appl. Crystallogr. 22261

Caglioti G, Paoletti A and Ricci F P 1958 Nucl. Instrum. Meth. 35223

Dollase W A 1986 J. Appl. Crystallogr. 19267

Fong D D, Stephenson G B, Streiffer S K, Eastman J A, Auciello O and Fuoss P H 2004 Science 3041650

Fu H X and Bellaiche L 2003 Phys. Rev. Lett. 91257601

Guinier A 1963 X-ray diffraction (SanFrancisco: W.H. Freeman)

Iakovlev S, Solterbeck C -H, Es-Souni M and Zaporojtchenko V 2004 Thin Solid Films 44650

Ishikawa K, Yoshikawa K and Okada N 1988 Phys. Rev. B37 5852

Jiang Q, Cui X F and Zhao M 2004 Appl. Phys. A - Mater. 78703

Jona F and Shirane G 1993 Ferroelectric crystals (New York: Dover Publications)

Kim S, Jun M -C and Hwang S-C 1999 J. Am. Ceram. Soc. 82289

Lang X Y and Jiang Q 2007 J. Nanopart. Res. 9595

Marshall W and Lovesey S W 1971 Theory of neutron scattering (Oxford: Clarendon Press)

Neves P P, Doriguetto A C, Mastelaro V R, Lopes L P, Mascarenhas Y P, Michalowicz A and Eiras J A 2004 J. Phys. Chem. B108 14840

Obmann P L and Glaubitt W 1997 J. Am. Ceram. Soc. 802658

Prince E 1982 Mathematical techniques in crystallography and material science (New York: Springer)

Prince 1985 in Structure and statistics in crystallography (ed.) A J C Wilson (Guilderland, NY: Adenine Press) pp. 95-108.

Ramesh R and Schlom D G 2002 Science 2961975

Rietveld H M 1988 Australian J. Phys. 41113

Rotella F J 1988 User's manual for Rietveld analysis of time-offlight neutron powder diffraction data at IPNS (USA: Argonne National Laboratory)

Sahu Niranjan, Panigrahi S and Kar Manoranjan $2010 \mathrm{~J}$. Adv. Powd. Technol. 22689

Tickoo R, Tandon R P, Hans V K, Bamzai K K and Kotru P N 2002 Mater. Sci. Eng. B94 1

Venkateswarlu P, Laha Apurba and Krupanidhi S B 2005 Thin Solid Films $\mathbf{4 7 4} 1$

Wiles D B and Young R A 1981 J. Appl. Crystallogr. 14 149

Wójcik K 1989 Ferroelectrics 995

Young R A 1996 The Rietveld method, International Union of Crystallography (New York: Oxford University Press)

Young R A and Wiles D B 1982 J. Appl. Crystallogr. 15 430

Zembilgotov A G, Pertsev N A, Kohlstedt H and Waser R 2002 J. Appl. Phys. 912247

Zhang S, Li H and Li M 2008 Mater. Letts 622438 